\title{
BMJ Open Hummingbird Study: a study protocol for a multicentre exploratory trial to assess the acceptance and performance of a digital medicine system in adults with schizophrenia, schizoaffective disorder or first-episode psychosis
}

To cite: Fowler JC, Cope N, Knights J, et al. Hummingbird Study: a study protocol for a multicentre exploratory trial to assess the acceptance and performance of a digital medicine system in adults with schizophrenia, schizoaffective disorder or first-episode psychosis. BMJ Open 2019;9:e025952. doi:10.1136/ bmjopen-2018-025952

- Prepublication history and additional material for this paper are available online. To view these files, please visit the journal online (http://dx.doi. org/10.1136/bmjopen-2018025952).

Received 13 August 2018

Revised 5 June 2019

Accepted 7 June 2019

Check for updates

(C) Author(s) (or their employer(s)) 2019. Re-use permitted under CC BY-NC. No commercial re-use. See rights and permissions. Published by BMJ.

For numbered affiliations see end of article.

Correspondence to

Dr J Corey Fowler;

corey.fowler@otsuka-us.com

\section{ABSTRACT}

Introduction In patients with schizophrenia, medication adherence is important for relapse prevention, and effective adherence monitoring is essential for treatment planning. A digital medicine system (DMS) has been developed to objectively monitor patient adherence and support clinical decision making regarding treatment choices. This study assesses the acceptance and performance of the DMS in adults with schizophrenia, schizoaffective disorder or first-episode psychosis and in healthcare professionals (HCPs).

Methods/Analysis This is a multicentre, 8-week, single-arm, open-label pragmatic trial designed using coproduction methodology. The study will be conducted at five National Health Service Foundation Trusts in the UK. Patients 18-65 years old with a diagnosis of schizophrenia, schizoaffective disorder or first-episode psychosis will be eligible. HCPs (psychiatrists, care coordinators, nurses, pharmacists), researchers, information governance personnel, clinical commissioning groups and patients participated in the study design and coproduction. Intervention employed will be the DMS, an integrated system comprising an oral sensor tablet coencapsulated with an antipsychotic, non-medicated wearable patch, mobile application (app) and web-based dashboard. The coencapsulation product contains aripiprazole, olanzapine, quetiapine or risperidone, as prescribed by the HCP, with a miniature ingestible event marker (IEM) in tablet. On ingestion, the IEM transmits a signal to the patch, which collects ingestion and physical activity data for processing on the patient's smartphone or tablet before transmission to a cloud-based server for viewing by patients, caregivers and HCPs on secure web portals or mobile apps.

Ethics and dissemination Approval was granted by London - City and East Research Ethics Committee (REC ref no 18/L0/0128), and clinical trial authorisation was provided by the Medicines and Healthcare products Regulatory Agency. Written informed
Strengths and limitations of this study

- This study was codeveloped with input from clinical practitioners, researchers, patients, caregivers, service managers, information governance teams and clinical commissioning groups to obtain information that will best serve all those involved in the treatment and care of patients with schizophrenia, schizoaffective disorder and first-episode psychosis.

- The digital medicine system (DMS) uses an integrated, user-friendly system to provide objective feedback on patient medication adherence and physical health parameters, such as activity and rest levels, as well as voluntary subjective entries regarding mood and rest quality, to help support treatment decisions and evaluation.

- Information from the DMS is made readily available to patients, caregivers (with the patient's consent) and physicians to support clinical decision making, proactive intervention and individualised care in near to real time.

- Possible limitations of this study are the small sample size $(n=60)$, limited generalisability to the broader pool of UK mental health patients and providers, and the relatively short 12-week timeframe.

- The DMS was not developed for all mental health patients, but for a subset of patients who realise they have difficulty with adherence and want to improve their status by self-monitoring, with potential for their healthcare professionals to make better clinical decisions based on objective data from the DMS.

consent will be obtained from every participant. The trial will be compliant with the International Council for Harmonisation of Technical Requirements for Pharmaceuticals for Human Use guidelines and the Declaration of Helsinki.

Trial registration number NCT03568500; EudraCT2017-004602-17; Pre-results. 


\section{INTRODUCTION}

Schizophrenia has an enormous social and economic burden worldwide, accounting for $1.1 \%$ of total disability-adjusted life-years and $2.7 \%$ of years lived with disability. ${ }^{12}$ In Europe, the economic impact of schizophrenia and other psychotic disorders and syndromes in 2010 was estimated at $€ 93.9$ billion. ${ }^{3}$ Because schizophrenia is a chronic disease, maintenance treatment is often necessary to prevent relapse and preserve quality of life over the long term. ${ }^{45}$ Although antipsychotic medications are effective, ${ }^{6-8}$ poor insight into schizophrenia can increase risk of medication non-adherence. ${ }^{9} 10$ In patients with schizophrenia, the rate of non-adherence to prescribed antipsychotics has been reported to be as high as $74 \% .{ }^{11}$ Not only is medication non-adherence burdensome to patients and their families, it also increases the likelihood of rehospitalisation and relapse ${ }^{12}$ and contributes substantially to healthcare costs. ${ }^{13}$ In 2005/2006, mean annual psychiatric inpatient and medication costs for patients with schizophrenia in England were estimated to be approximately $£ 3000$ and $£ 2000$ per patient, respectively. $^{14}$

Because medication adherence is important to relapse prevention in schizophrenia, ${ }^{15}$ effective adherence monitoring is an essential part of the treatment plan. Although there are various subjective methods for determining medication adherence, including patient surveys, adherence diaries and clinician ratings of patient symptoms and medication side effects, these methods have low validity. ${ }^{16}$ Blister packs and electronic medication bottle caps can provide an indicator of pill removal or bottle opening and have been shown to improve adherence, ${ }^{17} 18$ but neither technology provides an objective marker of pill ingestion or supports remote monitoring by healthcare professionals (HCPs). Optimal adherence monitoring and support for patients with schizophrenia requires a system that provides physicians with objective information during interim periods between patient visits.

The use of modern technology such as personal digital assistants, ${ }^{19}$ digital wristwatches, ${ }^{20}$ handheld computers ${ }^{21}$ and mobile phones ${ }^{22}$ to help people with schizophrenia dealing with their disease has shown success. A digital medicine system (DMS) has been developed as a drugdevice combination to objectively assess and report ingestion of prescription antipsychotics. ${ }^{23}$ The DMS has four main components: an ingestible sensor embedded within an inert tablet, a non-medicated wearable sensor (patch) worn by the patient, a mobile application (app) and a web-based dashboard. ${ }^{23}$ On interaction with stomach fluids, the ingestible sensor is activated and communicates with the wearable sensor, which sends a signal to a Bluetooth-paired mobile device where it can be viewed by patients or be subsequently viewed by HCPs and caregivers using secure mobile-based and cloud-based software. $^{23}$ The DMS also enables patients to share data on their activity and rest levels, as well as subjective data on mood and rest quality, which can be generated while the patient is engaged with the system. The intention of the
DMS is to encourage greater patient self-management and behaviour change while enabling caregivers and HCPs to provide better care and support both within and outside of office visits, further engaging patients in their ongoing disease management. The data are communicated to the psychiatrist that is connected to the patient on the DMS platform. Additionally, should the patient choose to share their data, they are able to invite additional healthcare providers, caregivers and/or family or friends. Recipients of the data, through a web-based, password-protected platform, are able to view these data and assist the patient with their treatment plan; however, HCPs can only access the portal for a specific patient once he/she has consented to give them access to their information in the system. It is envisioned that HCPs will be able to use these data to make more informed clinical decisions, such as whether individuals need dose adjustment, medication changes or conversations on lifestyle, adherence, or other parameters.

Previous results in the USA have shown in an openlabel, 8-week study that $78 \%(47 / 60)$ of patients and $72 \%$ (43/60) of HCPs reported being somewhat satisfied, satisfied or extremely satisfied with the DMS. ${ }^{24}$ Findings from another open-label study showed patients to be actively involved in their treatment, with $73.5 \%$ (36/49) using call centres for technological DMS support ${ }^{25}$; the rate of ingestion adherence in this study for the 15 patients with schizophrenia was $85.6 \% .^{25}$

The DMS from the current study (namely the ingestible sensor and the wearable sensor) are Conformité Européene (CE)-marked for use in Europe as class IIa medical devices (CE 559373), but studies in European mental health populations have not been performed. Therefore, the objective of this exploratory study is to assess the acceptance and performance of the DMS among adult patients with schizophrenia, schizoaffective disorder or first-episode psychosis and among HCPs from different care settings in the UK. We are particularly interested in assessing the acceptance of the digital medicine technology in individuals from different care settings. Acceptance will be assessed by study completion and feedback from subjects from patient satisfaction surveys. Furthermore, acceptance will also be evaluated by healthcare providers using the system; this will be assessed by how their clinical decisions altered while using the system and through HCP utility questionnaire evaluations.

In respect to performance, the study will be assessing multiple hardware and software from a varied population. Based on operational feedback of different phones and operating system and any technical troubleshooting that occurs, the study will be able to determine areas of the app that need to be enhanced to ensure that the app functions across multiple hardware and operating systems.

Previous studies performed using the DMS were conducted in relatively stable individuals with schizophrenia. For this study, we have broadened the inclusion criteria and will be assessing the technology in a range of 
clinical groups from different care settings, such as those individuals managed in the community or on specialised services, such as early intervention in psychosis services, to determine the performance in these different environments.

The study is not intended to measure and report or make any claims of adherence, but instead to report the observed ingestions recorded by the DMS. Our goal is to look at the impact of patients' improvements as a result of participation (eg, reduced need for follow-up care, knowledge of adherence to medication to help physicians decide whether patients are medication-compliant or require a long-acting injectable or other follow-up care), with the hypothesis that the DMS will reduce overall healthcare utilisation burden by optimising treatment decisions.

\section{METHODS AND ANALYSIS}

\section{Study design}

This is a multicentre, 8-week, single-arm, open-label trial to evaluate the acceptance and performance of the DMS in adult patients with schizophrenia, schizoaffective disorder or first-episode psychosis on an oral atypical antipsychotic medication (aripiprazole, olanzapine, quetiapine or risperidone) and in HCPs. Recruitment began the same day that we submitted to CT.gov, 25 May. However, we did not consent our first patient until 11 June and dosing occurred on 18 June. The study will take place at five institutions in the UK: Northumberland, Tyne and Wear National Health Service (NHS) Foundation Trust, Surrey and Borders Partnership NHS Foundation Trust, Oxford Health NHS Foundation Trust, South London and Maudsley NHS Foundation Trust, and Southern Health NHS Foundation Trust.

\section{Patient selection}

Patients for this exploratory study will be identified using database searches conducted at each study site per HCP discretion. Inclusion and exclusion criteria are provided in box 1 . The degree of clinical stability will be varied across participants who enrol. In short, a fully stable patient population will not be actively recruited, instead a range of clinical populations (crudely based on the Clinical Global Impression - Severity Scale) from different care settings will participate.

\section{Intervention}

The DMS is an integrated, four-component system comprising an oral coencapsulation (CoE) pharmacological sensor tablet, a Proteus Patch, a mobile app and the Otsuka medical dashboard software (figure 1). Each CoE product contains an approved antipsychotic medication (aripiprazole, olanzapine, quetiapine or risperidone) encapsulated with a miniature ingestible event marker (IEM) in tablet. HCPs will select the medication and dosage based on each patient's needs. On taking the medication, the IEM transmits a signal to the
Box 1 Inclusion and exclusion criteria

\section{Inclusion criteria.}

- Willing and able to give written informed consent and adhere to trial procedures.

- Able to read and understand English.

- Aged 18-65 years at the time of informed consent.

- Possessing a smartphone and able to use it to interact with the digital medicine system (DMS) application through robust and dependable cellular or wireless internet connections (Subjects should have WiFi at home and/or at work, or at the very least have access to free WiFi hot spots. Alternatively, subjects should have a sufficient data plan from their mobile provider and/or coverage on their phone. Such assessments are made during the screening of potential subjects.).

- Cooperative, able to ingest oral medication, willing to complete aspects of the trial and capable of reporting adverse events (AEs).

- Clinical diagnosis of schizophrenia, schizoaffective disorder (defined by the International Classification of Diseases, Tenth Revision, codes F20 and F25) or first-episode psychosis based on case note review.

- Prescription for aripiprazole, olanzapine, quetiapine or risperidone.

- Fulfils $\geq 1$ of the following:

- Discharge from a hospital admission (within 7 days of discharge) to an acute intervention team.

- Referral to an acute intervention team before hospital admission.

- Referral from an acute intervention team to a community team.

- Managed by community services.

- Inclusion within early intervention case load ( $<3$ years from initial symptoms).

- Healthcare professional (HCP) determines the patient would benefit.

- General medical condition does not pose additional risk.

- Skin on the anterior chest above the lower edge of the rib cage is free of any dermatological problem.

\section{Exclusion criteria.}

- Any disorder that may affect the patient's ability to participate in the trial or interact with a smartphone.

- Likely to be incapable of using the DMS technology, even with assistance.

- History or evidence of a medical condition that would expose the patient to undue risk of an AE.

- Known allergy to adhesive tape or any component of the patch or coencapsulation product.

- Current incarceration in prison system.

- Hospitalisation at the time of screening due to mental or physical illness.

- HCP recommendation to not participate.

- Aversion to taking gelatin capsules.

- Women who are breast feeding, pregnant or plan to become pregnant.

non-medicated patch that is attached to the patient's skin. The patch collects IEM ingestion and physical activity data, which are then processed by the Patch Analytics Block on the patient's smartphone. Processed data are transferred through the app on the smartphone and sent to a cloud-based server for patient, caregiver and HCP viewing on Otsuka medical software through web portals or mobile apps. Patients will have access to the Otsuka Patient Application, caregivers will have access (with patient consent) to the Otsuka Caregiver Web Portal, and 


\section{Digital Medicine overview \\ Provides objective and unbiased data to support clinical decision making}

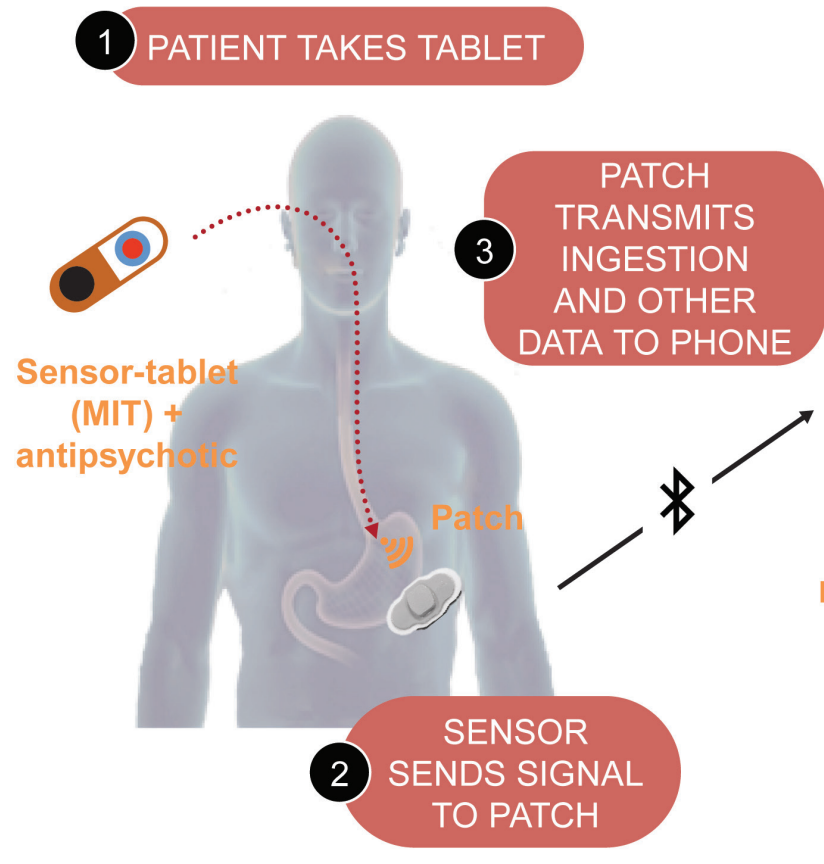

*Except for the first ingestion

${ }^{\dagger}$ Caregiver defined as patient-consented individual privy to DM data streams

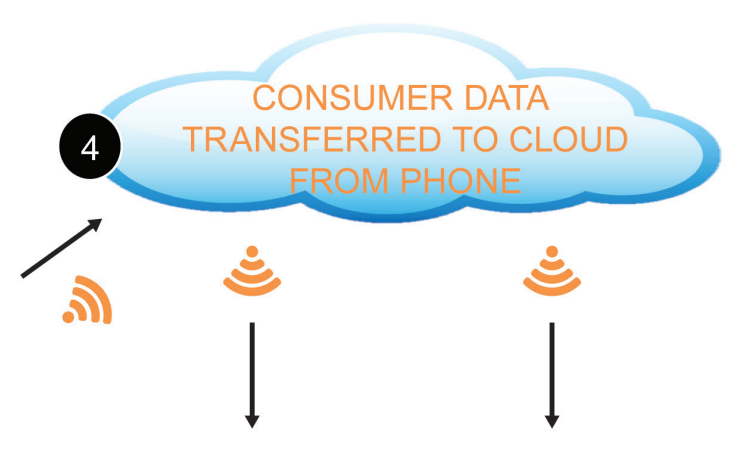

Patient Smartphone (Pre-Cloud Data Transfer)

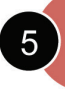

PATIENT/HCP/CAREGIVER* VIEW DATA VIA PHONE/DASHBOARD
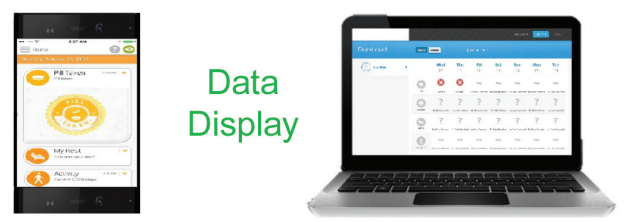

Mobile App for Patient Smartphone (Post-Cloud Data Transfer)

Figure 1 Digital medicine system: coencapsulated antipsychotic medication with miniature ingestible event marker in tablet (MIT) and compatible medical device. Image reprinted from an article in npj Digital Medicine. ${ }^{32} \mathrm{DM}$, digital medicine; HCP, healthcare professional.

HCPs will have access to the Otsuka Healthcare Provider Web Portal.

\section{Patient and public involvement statement Coproduction}

This study was designed using coproduction methodology involving NHS staff and patients (figure 2). Coproduction in this protocol is the involvement of people with lived experience of mental illness (diagnosed or otherwise) as equal partners alongside other healthcare stakeholders in the design and contribution to the protocol. The lead site, Southern Health NHS Foundation Trust, held five coproduction workshops, and a subset of these workshops were repeated at other study sites to elicit feedback on the study design. At least two of these workshops involved patients. The discussions from the workshops addressed pharmacy items for the CoE product; HCP recruitment strategies; and protocols for identification, recruitment and interactions with suitable patients and their general practitioners. Throughout the various workshops at the different sites, participants included clinicians, pharmacists, researchers, information governance (IG) personnel (refers to the way in which the NHS handles, stores and processes information, in particular personal and sensitive information relating to patients and employees; it was vital to ensure that IG individuals were happy with the privacy and storage features of the DMS), psychiatrists, care coordinators, nurses, information technology personnel, clinical commissioning groups (clinically led groups within the NHS that are responsible for the planning and commissioning of healthcare services for their local area) and patients (figure 2).

\section{Patient involvement}

The patient and public involvement and service user lead from two study sites (Surrey and Borders Partnership NHS Foundation Trust, and Northumberland, Tyne and Wear NHS Foundation Trust) engaged service users to participate in a patient focus group. The objective of the focus groups was to obtain feedback on the app technology and assess the completion of specific app tasks. The groups identified issues that may have prevented the completion of key tasks and whether greater explanation would be needed, for instance in ensuring the app could send notifications to patients. Furthermore, general feedback on colour and language was also obtained. 


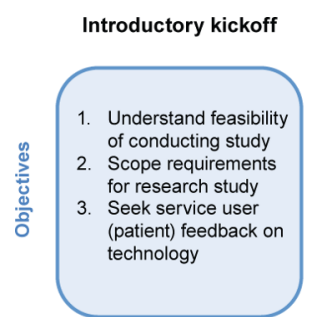

Service user DMS feedback
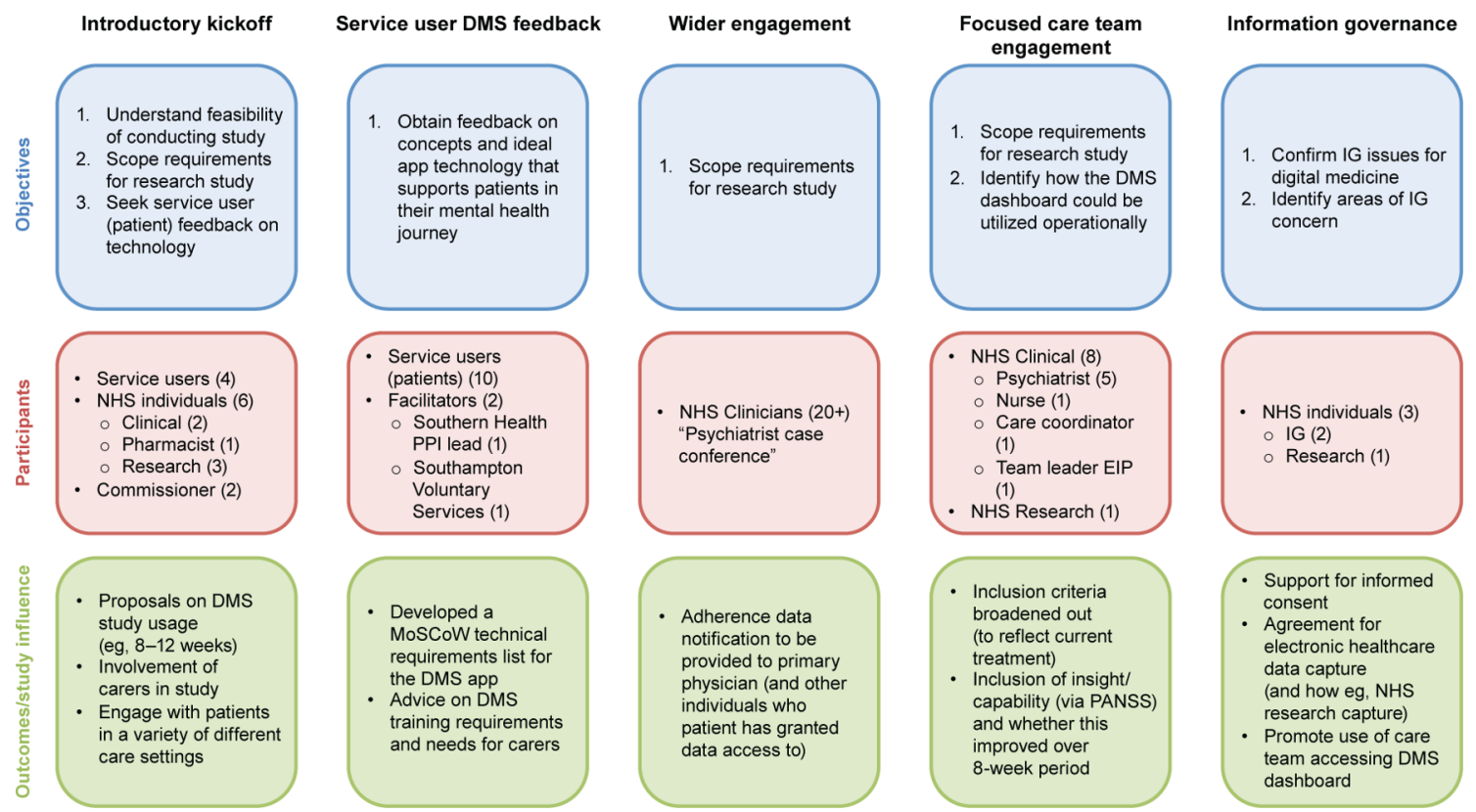

Figure 2 Description of coproduction workshops with Southern Health NHS Foundation Trust. DMS, digital medicine system; EIP, early intervention in psychosis; IG, information governance; MoSCoW, must have, should have, could have, won't have; NHS, National Health Service; PANSS, Positive and Negative Syndrome Scale; PPI, patient and public involvement programme.

\section{Procedures}

The study will comprise three phases: a screening phase of up to 7 days, an 8-week assessment phase and a 2-week safety follow-up (figure 3). Screening and baseline may occur at a single visit or at two visits separated by up to 7 days. At the screening/baseline visit, patients providing informed consent will receive patches, $\mathrm{CoE}$ product and other supplies, and undergo training by the HCPs on required and correct use of the DMS. HCPs will confirm proper patch application to the skin and pairing of the patch with the smartphone app when patients commence their usage of the DMS, the so-called on boarding.
These individuals will either be psychiatrists or research assistants for the site. During time in between the only required site visits at weeks 4 and 8 , patients will perform patch changing themselves and be guided, if required, through videos contained within the app. There is a freephone technical support line to assist individuals should they wish. Patients will be instructed to wear the patch continuously, replacing it every 7 days or as needed during the assessment period. An integrated call centre will be available to patients for technical support regarding use of the DMS.

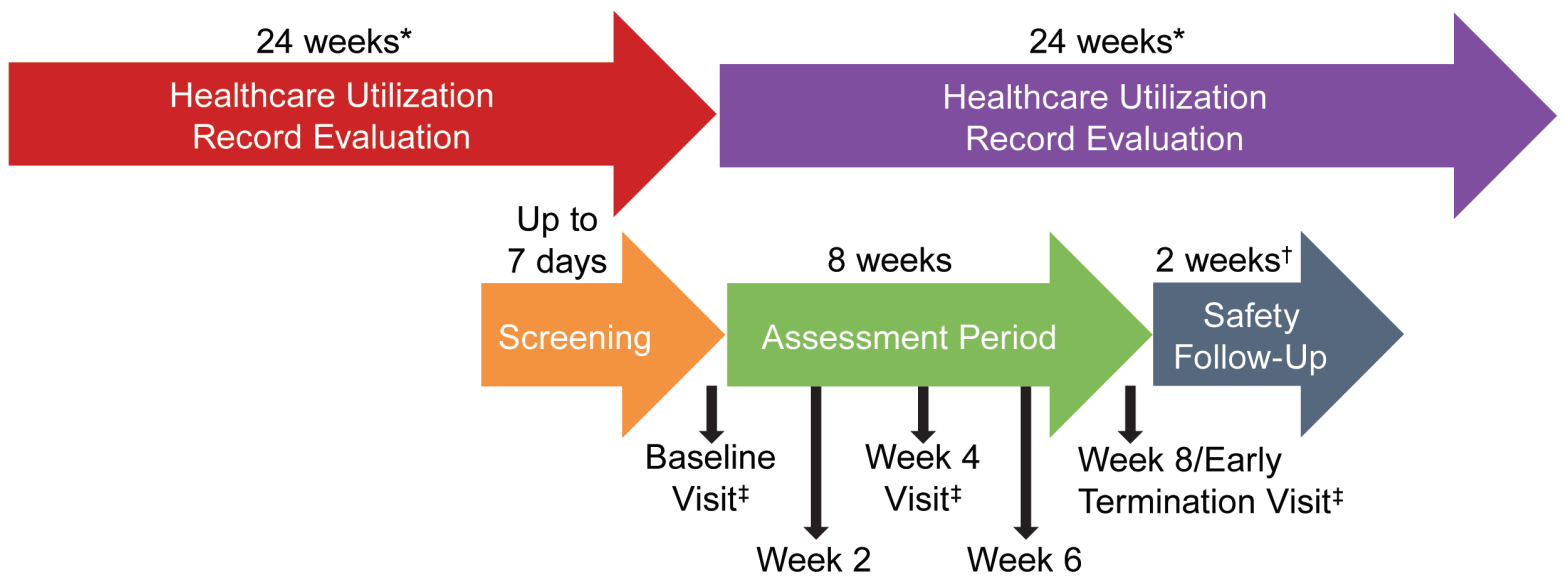

Healthcare professionals (HCPs) will review dashboard at weeks 2, 4, 6, and 8 and make treatment changes at their discretion. *Healthcare utilization will be recorded 24 weeks before and after baseline visit (for a total of 48 weeks).

tSafety follow-up phone call will occur 2 weeks after week 8/early termination visit.

fPatient visits will occur at baseline, week 4, and week 8/early termination. Other visits will be at the discretion of the HCP.

Figure 3 Study design. 
During the subsequent 8-week assessment period, patients will visit their HCP at weeks 4 and 8 (or at early termination) for clinical evaluation and review of DMS data. At the week 4 visit, patients will also obtain a prescription refill. HCPs will be required to monitor and review DMS data at least every 2 weeks, with the option of requesting additional patient visits as needed. HCPs will also have the ability to modify the patient's treatment plan at their discretion. Safety and tolerability will be assessed on an ongoing basis throughout the assessment period and during the subsequent 2-week safety follow-up, during which time a follow-up telephone call will be made to each study participant.

Healthcare utilisation record evaluation will occur continuously beginning at the baseline/screening visit and will span a period ranging from 24 weeks before baseline/screening to 24 weeks after screening. All hospital admissions will be recorded and categorised as 'planned', 'unplanned', 'related' or 'unrelated' to psychiatric illness. Characteristics of each HCP encounter will also be recorded, including the nature of the contact, HCP role, whether the visit was planned or unplanned, whether or not the contact was initiated as a result of the DMS, and any medication titration, adherence counselling, education or lifestyle coaching that occurred.

\section{Outcomes}

The primary endpoint is the proportion of days with good patch coverage during the assessment period, which will be defined as having $\geq 80 \%$ patch data available or IEMs detected within each day of the assessment period. The secondary endpoint is ingestion adherence, defined as the proportion of detected IEMs to the total expected IEMs ingested on the assessment days that showed good patch coverage.

The following exploratory endpoints will also be included:

- The proportion of time during the assessment period that patients wear their patch.

- Engagement and satisfaction of patients, HCPs and caregivers as determined using the Subject Usability and Satisfaction Scale (see online supplementary figure 1 ), the Physician Utility Survey (see online supplementary figure 2) and the Caregiver/Support Person Involvement Scale (see online supplementary figure $3)$.

- Personal and social functioning as assessed using the Personal and Social Performance Scale (see online supplementary figure 4).

- Patient activation as assessed using the Patient Activation Measure-Mental Health Scale (see online supplementary figure 5).

- The proportion of days that patients and HCPs use the app and dashboard, respectively.

- The proportion of ingested IEM tablets registered on the digital health data server of the total expected IEM tablets ingested.
- Safety variables will include the frequency and severity of serious adverse events and device-related non-serious adverse events, suicidality and any product quality complaints that may arise. Suicidality will be determined by face-to-face risk assessment according to each study site's standard operating procedure.

\section{Statistical analysis}

A sample size of 60 patients was determined based on an anticipated $25 \%$ discontinuation rate, such that $\geq 45$ patients would complete the 8-week assessment period. The study described is a feasibility study with no comparisons and no formal power calculations. The sample size was chosen to contain roughly 20 patients per indication and align with historical studies performed in the USA. The discontinuation rate is assumed based on similar discontinuations for other psychiatry studies and the fact that an actively clinically stable population is not being recruited. Data will be analysed in the intent-totreat population (ie, all patients who enter the trial and use the DMS). All data will be analysed using descriptive statistics. Continuous variables will be summarised using mean, median, range and SD. Categorical variables will be summarised using frequency distributions.

\section{Data protection}

Before initiating participation, informed consent will be obtained from all patients and caregivers. Health information will be de-identified to the fullest possible extent. However, some identifiers or sensitive health information cannot be stripped (eg, psychiatric medical history, participation in a care programme approach, presence of a community treatment order, employment status, disabilities, housing arrangements or armed forces history). Health information will be used to develop and improve the DMS app and user experience. The DMS and associated third-party vendors are compliant with the General Data Protection Regulation (GDPR), and all accessed health information will be maintained in the strictest confidence and in compliance with the GDPR. Data from the DMS are encrypted in activity and rest and will be accessed only by role-specific individuals for discrete time periods and functions (eg, technical troubleshooting). All data will be completely anonymised for graphical, statistical and publication purposes.

Research staff from NHS sites will store consent documents, demographic forms and receipts for reimbursement of travel in locked filing cabinets to which only research staff will have access. Accessing of health data will be compliant with the IG policies of each participating NHS Trust.

\section{Ethical considerations}

Written informed consent will be obtained from every participant. Ethical issues will be related to the identification and recruitment of patients, informed consent and data protection arrangements. The trial will be conducted in compliance with the International Council 
for Harmonisation of Technical Requirements for Pharmaceuticals for Human Use guidelines and the Declaration of Helsinki.

\section{Dissemination of study results}

The study results will be disseminated through peer-reviewed publications, national and international conference presentations, and formal clinical trial repositories (eg, ClinicalTrials.gov). The five participating NHS sites will be invited to a results launch event. Results will be shared with individuals who participated in the workshops via the respective Trusts. Additionally, follow-up workshops are planned to capture further feedback on the next steps for scaling the DMS technology.

\section{DISCUSSION}

Patient adherence to antipsychotic medication is a crucial component of successful maintenance therapy for schizophrenia. Knowledge of patient adherence in the absence of positive treatment outcome is also essential. Subjective means for assessing medication adherence such as clinician evaluation and patient self-report have low validity, ${ }^{16}$ and most can only be administered during patient-HCP visits. Digital medicine offers a means to monitor patient adherence and obtain objective data throughout treatment, including periods between office visits, which may yield more accurate data. For example, a study evaluating non-adherence rates for antipsychotic medication based on electronic medication bottle caps, which report when pill bottles have been opened, ${ }^{18}$ showed a $57 \%$ non-adherence rate, much higher than that estimated by patient $(5 \%)$ and provider $(7 \%)$ report. ${ }^{18}$ Electronic medication bottle caps are used as the current gold standard surrogate for 'objective' adherence data. Few reports in this space exist and the need for more robust objective adherence data is supported through this discrepancy and the limitations of electronic medication bottle caps as an 'objective' measure, given it only measures an intermediate step in the ingestion process. This study shows the potential of digital medicine to provide continuous objective data that can inform and improve patient adherence and assist in clinical decision making. The DMS improves on approaches such as electronic medication caps by providing an objective marker of ingestion and not merely a surrogate in addition to other objective and subjective data.

Patient discontinuation is a considerable problem in studies of digital health technology. ${ }^{26}$ Studies of early digital health systems for maintenance and counselling of mental disorders reported low completion rates. For example, a study evaluating a 12-week, web-based cognitive behavioural therapy intervention for panic disorder had a $1 \%(12 / 1161)$ completion rate, ${ }^{27}$ and a study evaluating a publicly accessible five-module, web-based cognitive behavioural therapy programme for anxiety and depression had a $0.5 \%$ completion rate $(97 / 19607){ }^{28}$ Completion rates may be particularly low in patients with more severe negative symptoms. ${ }^{22}$ Despite these historically poor completion rates in studies of digital health platforms, results of a meta-analysis indicate that more than $50 \%$ of patients favour managing their mental health through the use of mobile health technology, ${ }^{29}$ and the use of mobile devices for health management is viewed by patients as normalised and non-stigmatising. ${ }^{30}$ The integrated DMS described here was developed to leverage the advantages of digital technology to positively influence adherence in patients with schizophrenia.

The DMS is a multimodal, user-friendly system that provides medication reminders and measures patient ingestion of prescribed antipsychotic medications. The CoE product, in particular, advances the ability to confirm medication ingestion and to assess adherence on a continuous basis, which can inform whether uncontrolled symptoms may be explained by non-adherence. Information from the DMS is made readily available to patients, caregivers (with the patient's consent) and physicians for supported decision making, proactive intervention and individualised care. The DMS also provides adherence feedback electronically to the patient, HCP and/ or caregiver. The DMS provides this feedback discreetly, through user-owned and operated apps and a patch that is not readily visible as it is worn on the torso underneath clothing, reducing any potential stigmatisation if it (the patch) was visible. The DMS also provides feedback on activity and rest levels, which is important given that maintenance of a healthy lifestyle can help address the problems of weight gain and obesity that are related to both medication side effects and sedentary lifestyles. ${ }^{31}$ Furthermore, shifts in activity levels may be indicative of altered patient disposition.

This ongoing study was designed using coproduction methodology to incorporate input from patients, caregivers, psychiatrists, pharmacists, nurses and researchers. For all five study sites, a minimum of two engagement/ set-up meetings were conducted and two specific patient focus groups were held to guide protocol development and app design. Although a PRagmatic Explanatory Continuum Indicator Summary 2 (PRECIS-2) assessment tool was not used during the design of the study, the authors feel that based on a retrospective PRECIS-2 analysis (refer to online supplementary material for this analysis), this study does meet the criteria for a pragmatic study. The exploratory data collected in this study, although limited to a small number of patients, will help provide a better understanding of the ease of use for patients, HCPs and caregivers to inform the development of future software or hardware iterations.

Given the level of unfamiliarity with the DMS, conducting clinical trials in this space requires a higher level of stakeholder management and alignment, especially when the trial is being held in a new environment. Whether it is a new country, a new healthcare system or a new set of investigators, formal buy-in and input is critical to participation. The protocol outlines one way of managing and aligning stakeholders in such an 
environment-the UK mental health system. Additionally, and increasingly important within mental health services and interventions in the UK, is the involvement of end users, the so-called service users who have lived experience of mental health. The methods paper describes a robust engagement strategy using such individuals in mental health research.

Digital health interventions require significant clinician and patient engagement. The protocol describes an approach to ensure that service users of a digital medicine intervention can assist with protocol design and system input, for example, approach and appropriate language.

\section{CONCLUSION}

In conclusion, this study will examine the usability and acceptance of the DMS by patients with schizophrenia, schizoaffective disorder or first-episode psychosis and by caregivers and HCPs in different care settings. The study was coproduced through the engagement of all stakeholders and, if successful, could be used to support DMS usage in larger cohorts within the UK and Europe.

\section{Author affiliations}

${ }^{1}$ Otsuka Pharmaceutical Development \& Commercialization, Inc., Princeton, New Jersey, USA

${ }^{2}$ Otsuka Pharmaceutical Europe, Wexham, UK

${ }^{3}$ Southern Health NHS Foundation Trust, Southampton, UK

${ }^{4}$ Otsuka Europe Development and Commercialisation, Wexham, UK

Acknowledgements The authors thank patient advisers and NHS staff from all five participating sites (Oxford Health NHS Foundation Trust, Northumberland, Tyne and Wear NHS Foundation Trust, Southern Health NHS Foundation Trust, South London and Maudsley NHS Foundation Trust, and Surrey and Borders Partnership NHS Foundation Trust) for their contributions to the study methodology. Editorial support for the development of this manuscript was provided by Eric Comeau, PhD, at C4 MedSolutions (Yardley, Pennsylvania, USA), a CHC Group company.

Contributors JCF, NC, JK, PP, AM, TP-S and SR contributed to study conception and design, as well as data collection, analysis and interpretation. JCF, NC, JK, PP, AM, TP-S and SR were responsible for review and critical revision of the manuscript. JCF, NC, JK, PP, AM, TP-S and SR gave final approval to submit for publication.

Funding This study is supported by Otsuka Pharmaceutical Development \& Commercialization, Inc. Editorial support was funded by Otsuka Pharmaceutical Development \& Commercialization, Inc.

Competing interests JCF, JK and TP-S are employees of Otsuka Pharmaceutical Development \& Commercialization. NC is an employee of Otsuka Pharmaceutical Europe. AM is an employee of Otsuka Europe Development and Commercialisation. SR has received honoraria from Otsuka and Lundbeck for educational sessions.

\section{Patient consent for publication Not required.}

Ethics approval Ethics approval for this study was obtained from London - City and East Research Ethics Committee (REC ref no 18/L0/0128), and clinical trial authorisation was provided by the Medicines and Healthcare products Regulatory Agency.

Provenance and peer review Not commissioned; externally peer reviewed.

Open access This is an open access article distributed in accordance with the Creative Commons Attribution Non Commercial (CC BY-NC 4.0) license, which permits others to distribute, remix, adapt, build upon this work non-commercially, and license their derivative works on different terms, provided the original work is properly cited, appropriate credit is given, any changes made indicated, and the use is non-commercial. See: http://creativecommons.org/licenses/by-nc/4.0/.

\section{REFERENCES}

1. World Health Organization. The Global Burden of Disease: 2004 update. 2008. http://www.who.int/healthinfo/global_burden_disease/ GBD_report_2004update_full.pdf?ua=1 [Accessed 29 June 2018].

2. Rathod S, Garner C, Griffiths A, et al. Protocol for a multicentre study to assess feasibility, acceptability, effectiveness and direct costs of TRlumPH (Treatment and Recovery In PsycHosis): integrated care pathway for psychosis. BMJ Open 2016;6:e012751.

3. Gustavsson A, Svensson M, Jacobi F, et al. Cost of disorders of the brain in Europe 2010. Eur Neuropsychopharmacol 2011;21:718-79.

4. Hasan A, Falkai P, Wobrock T, et al. World Federation of Societies of Biological Psychiatry (WFSBP) guidelines for biological treatment of schizophrenia, part 2: update 2012 on the long-term treatment of schizophrenia and management of antipsychotic-induced side effects. World J Biol Psychiatry 2013;14:2-44.

5. Kreyenbuhl J, Dixon LB, McCarthy JF, et al. Does adherence to medications for type 2 diabetes differ between individuals with vs without schizophrenia? Schizophr Bull 2010;36:428-35.

6. Isett KR, Burnam MA, Coleman-Beattie B, et al. The state policy context of implementation issues for evidence-based practices in mental health. Psychiatr Serv 2007;58:914-21.

7. Ismail Z, Peters-Strickland T, Miguelez M, et al. Aripiprazole Once-Monthly in the Treatment of Acute Psychotic Episodes in Schizophrenia: Post Hoc Analysis of Positive and Negative Syndrome Scale Marder Factor Scores. J Clin Psychopharmacol 2017;37:347-50.

8. Potkin SG, Loze JY, Forray C, et al. Multidimensional Assessment of Functional Outcomes in Schizophrenia: Results From QUALIFY, a Head-to-Head Trial of Aripiprazole Once-Monthly and Paliperidone Palmitate. Int J Neuropsychopharmacol 2017;20:40-9.

9. Rathod S, Kingdon D, Smith P, et al. Insight into schizophrenia: the effects of cognitive behavioural therapy on the components of insight and association with sociodemographics-data on a previously published randomised controlled trial. Schizophr Res 2005;74:211-9.

10. Sendt KV, Tracy DK, Bhattacharyya S. A systematic review of factors influencing adherence to antipsychotic medication in schizophreniaspectrum disorders. Psychiatry Res 2015;225:14-30.

11. Lieberman JA, Stroup TS, McEvoy JP, et al. Effectiveness of antipsychotic drugs in patients with chronic schizophrenia. $N$ Engl $J$ Med 2005;353:1209-23.

12. Morken G, Widen JH, Grawe RW. Non-adherence to antipsychotic medication, relapse and rehospitalisation in recent-onset schizophrenia. BMC Psychiatry 2008;8:32.

13. Cutler RL, Fernandez-Llimos F, Frommer M, et al. Economic impact of medication non-adherence by disease groups: a systematic review. BMJ Open 2018;8:e016982.

14. McCrone P, Dhanasiri S, Patel A, et al. Paying the Price: The Cost of Mental Health Care in England to 2026. London: King's Fund, 2008.

15. Gray R, Bressington D, Ivanecka $A$, et al. Is adherence therapy an effective adjunct treatment for patients with schizophrenia spectrum disorders? A systematic review and meta-analysis. BMC Psychiatry 2016;16:90.

16. Haddad PM, Brain C, Scott J. Nonadherence with antipsychotic medication in schizophrenia: challenges and management strategies. Patient Relat Outcome Meas 2014;5:43-62.

17. Gutierrez PM, Wortzel HS, Forster JE, et al. Blister Packaging Medication Increases Treatment Adherence in Psychiatric Patients. $J$ Psychiatr Pract 2017;23:320-7.

18. Byerly MJ, Thompson A, Carmody T, et al. Validity of electronically monitored medication adherence and conventional adherence measures in schizophrenia. Psychiatr Serv 2007;58:844-7.

19. Ben-Zeev D, McHugo GJ, Xie H, et al. Comparing retrospective reports to real-time/real-place mobile assessments in individuals with schizophrenia and a nonclinical comparison group. Schizophr Bull 2012;38:396-404.

20. Oorschot M, Lataster T, Thewissen V, et al. Mobile assessment in schizophrenia: a data-driven momentary approach. Schizophr Bull 2012;38:405-13.

21. Kimhy D, Delespaul P, Corcoran C, et al. Computerized experience sampling method (ESMc): assessing feasibility and validity among individuals with schizophrenia. J Psychiatr Res 2006;40:221-30.

22. Granholm E, Ben-Zeev D, Link PC, et al. Mobile Assessment and Treatment for Schizophrenia (MATS): a pilot trial of an interactive text-messaging intervention for medication adherence, socialization, and auditory hallucinations. Schizophr Bull 2012;38:414-25.

23. Rohatagi S, Profit D, Hatch A, et al. Optimization of a Digital Medicine System in Psychiatry. J Clin Psychiatry 2016;77:e110 1-e1107.

24. Peters-Strickland T, Pestreich L, Hatch A, et al. Usability of a novel digital medicine system in adults with schizophrenia treated with 
sensor-embedded tablets of aripiprazole. Neuropsychiatr Dis Treat 2016;12:2587-94.

25. Kopelowicz A, Baker RA, Zhao C, et al. A multicenter, open-label, pilot study evaluating the functionality of an integrated call center for a digital medicine system to optimize monitoring of adherence to oral aripiprazole in adult patients with serious mental illness. Neuropsychiatr Dis Treat 2017;13:2641-51.

26. Eysenbach G. The law of attrition. J Med Internet Res 2005;7:e11.

27. Farvolden $P$, Denisoff E, Selby $P$, et al. Usage and longitudinal effectiveness of a Web-based self-help cognitive behavioral therapy program for panic disorder. J Med Internet Res 2005;7:e7.

28. Christensen $\mathrm{H}$, Griffiths $\mathrm{KM}$, Korten AE, et al. A comparison of changes in anxiety and depression symptoms of spontaneous users and trial participants of a cognitive behavior therapy website. J Med Internet Res 2004;6:e46.

29. Firth J, Cotter J, Torous J, et al. Mobile phone ownership and endorsement of "mHealth" among people with psychosis: a metaanalysis of cross-sectional studies. Schizophr Bull 2016;42:448-55.

30. Palmier-Claus JE, Rogers A, Ainsworth J, et al. Integrating mobilephone based assessment for psychosis into people's everyday lives and clinical care: a qualitative study. BMC Psychiatry 2013;13:34.

31. Gossage-Worrall R, Holt RI, Barnard K, et al. STEPWISE - STructured lifestyle Education for People Wlth SchizophrEnia: a study protocol for a randomised controlled trial. Trials 2016;17:475.

32. Knights J, Heidary Z, Peters-Strickland T, et al. Evaluating digital medicine ingestion data from seriously mentally ill patients with a Bayesian Hybrid Model. NPJ Digit Med 2019;2. 